\title{
Temperature Effects in Liquid Cell Transmission Electron Microscopy
}

\author{
Frances Ross
}

\author{
Massachusetts Institute of Technology, Cambridge, Massachusetts, United States
}

Liquid cell transmission electron microscopy provides an opportunity for spatially and temporally resolved imaging of materials and processes in water and other liquids. The increasing breadth of liquid cell applications [1] is in part driven by experimental advances that enable new stimuli to be applied to the sample. A particularly promising direction for experimental development is the incorporation of temperature control into liquid cell experiments. Changes in temperature can be used to accelerate materials reactions such as nanocrystal nucleation and growth, or to trigger phase transformations such as ice or bubble formation [2]. Making the most of this new capability requires combining it with other stimuli and measuring or modeling the local environment of the sample as accurately as possible. We are particularly excited by the combination of temperature control with electrochemical control because of the fundamental importance of temperature to many electrochemical processes. For example, operation over a broad temperature range is a key requirement for battery designs intended for grid-scale storage [3], and temperature is controlled in material processing to set the rate of galvanic displacement or electrochemical etching. Here we discuss several aspects of liquid cell microscopy of temperaturecontrolled electrochemical processes. Figure 1 shows one particular strategy for simultaneous electrochemical and temperature control. Two electrically isolated electrodes are patterned onto a chip that has a resistive heater embedded within the electron transparent window, Figure 1a. The areas of the electrodes that are directly over the heater element experience a temperature rise when current passes through the heater, Figure 1b. The temperature distribution can be quantified using finite element modeling. Temperature-dependent electrochemical deposition using this type of chip is shown in Figure 1c. As expected, deposition of $\mathrm{Cu}$ from acidified copper sulphate solution occurs more rapidly at elevated temperature. Similarly, we find that electrochemically driven etching of bimetallic nanoparticles occurs at lower potential when the temperature is raised. In all liquid cell experiments the sample experiences an extreme environment due to the energy deposited by the imaging beam. Estimates for conventional TEM conditions [4] suggest that the dose rate to the water in a liquid cell sample is over $10^{7} \mathrm{~Gy} / \mathrm{s}$. This is seven orders of magnitude higher than the dose rates generated by common radiation sources and four orders higher than the dose rate estimated at the wall of the nuclear reactor ITER [5]. The water in the liquid cell undergoes radiolysis: radicals are generated, the $\mathrm{pH}$ changes, and radiolytic hydrogen and oxygen molecules may nucleate gas bubbles [4]. To assess temperature effects on radiolysis in TEM we note that virtually every parameter important in a radiolysis simulation is expected to change with temperature. This includes the formation rate of the radiolysis products, or $G$ values, and the rates of the reactions between radiolysis species, or $\mathrm{k}$ values, both of which are generally predicted to increase with temperature [6]. Since the steady state concentration of any radiolysis species scales as production / reaction rate $\mathrm{x}$ scavenger concentration, the increase in reaction rates means the main scavengers consume species like hydrated electrons more quickly, reducing the steady state concentrations. Diffusion coefficients are linked to viscosity and therefore also expected to depend on temperature [7, 8]. Finally, the solubilities of the gas species are expected to decrease as the temperature increases [9]. Overall, these changes suggest a complex dependence on temperature for chemical changes and electron beam-driven processes. But for particular physical phenomena other effects may be even more important: for example, in crystal nucleation and growth processes, activation energies, surface energies, solubility and critical nucleus size will lead to a temperature dependence. We suggest that liquid cell microscopy can provide unique insights 
and improved quantification of temperature effects with appropriate experimental and computational strategies.
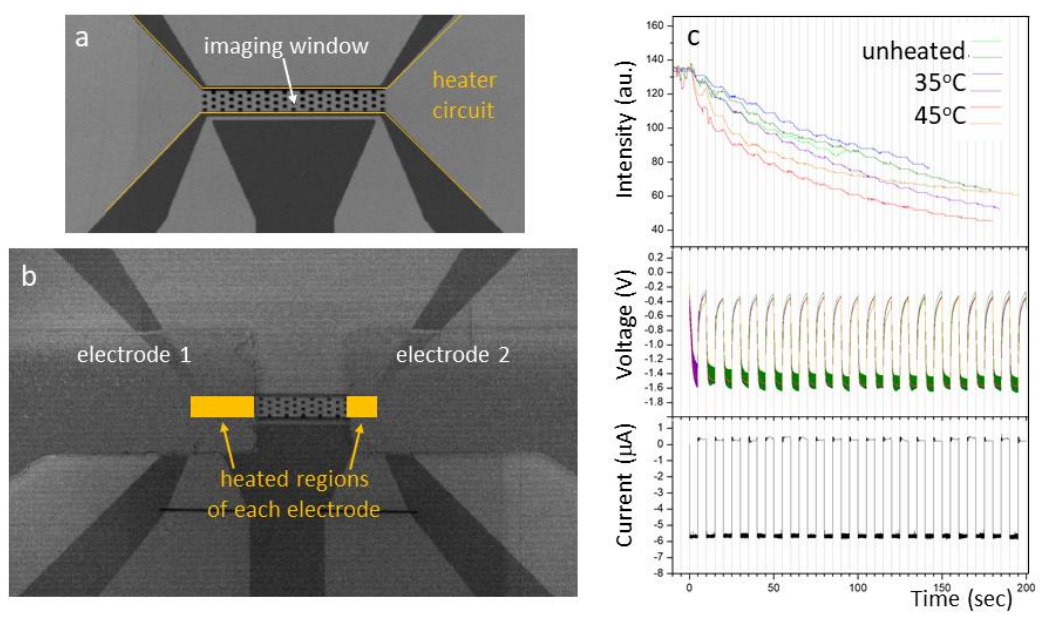

Figure 1. A proof of principle experiment showing simultaneous temperature and electrochemical control. (a) A liquid cell chip with Pt resistance heater [10] imaged in SEM; (b) Electrodes added over the heater using a shadow mask. Only a small portion of each electrode (yellow box) is heated. (c) Pulsed galvanostatic deposition of copper from $0.1 \mathrm{M} \mathrm{CuSO} 4+0.18 \mathrm{M}$ H2SO4. Intensity averaged over a $200 \mathrm{x}$ $200 \mathrm{~nm}$ region of the electrode, current and voltage are shown during deposition at different temperatures. As expected, higher deposition rate (lower intensity for the same charge passed) is observed at the heated areas of the electrode.

References [1] F. M. Ross, Science 350, aaa9886 (2016) [2] S. J. Dillon and X. Chen, Temperature control in liquid cells for TEM, Ch. 6 , in Liquid Cell Electron Microscopy, ed. F. M. Ross, Cambridge University Press (2016) [3] P. C. Butler, P. A. Eidler, P. G. Grimes, S. E. Klassen and R. C. Miles, Zinc / Bromine Batteries, Ch. 39, in Handbook of Batteries, ed. D. Linden and T. B. Reddy, McGraw-Hill (2001) [4] N. M. Schneider, M. M. Norton, B. J. Mendel, J. M. Grogan, F. M. Ross and H. H. Bau, J. Phys. Chem. C 118, 22373 (2014) [5] https://crppwww.epfl.ch/ weisen/Cours_fichiers/ITER\%20DIAGNOSTICS.pdf, accessed Feb 2020 [6] A. J. Elliot, M. P. Chenier and D. C. Ouellette, Can. J. Chem. 68, 712 (1990) [7] T. Baştuğ and S. Kuyucak, Chem. Phys. Lett. 408, 84 (2005) [8] M.-A. Hervé du Penhoat, T. Goulet, Y. Frongillo, M.-J. Fraser, P. Bernat and J.-P. Jay-Gerin, J. Phys. Chem. A 104, 11765 (2000) [9] B. Ambrožič, A. Prašnikar, N. Hodnik, N. Kostevšek, B. Likozar, K. Žužek Rožman and S. Šturm, Chem. Sci. 10, 8735 (2019) [10] http://hummingbirdscientific.com/products/liquid-heating/, accessed Jan 2020 